\section{La interpelación de la Reforma Universitaria a la agenda de la Red Nacional de Extensión Universitaria argentina}

Juan Pablo Itoiz

Secretario de Extensión

Universidad Nacional del Noroeste

de Buenos Aires, Argentina.

Coordinador Nacional Red de

Extensión (REXUNI), Consejo

Interuniversitario Nacional (CIN)

juanpabloitoiz@unnoba.edu.ar
A 100 años de la Reforma Universitaria de 1918 /

Desafíos de gestión

RECEPCIÓN: 29/06/17

ACEPTACIÓN FINAL: 24/09/17

\section{Resumen}

El presente artículo propone reflexionar acerca de los recorridos y desafíos que ha enfrentado y enfrenta la extensión universitaria como función sustantiva de las instituciones de educación superior de gestión pública. Este tránsito es continuo y fluido, propio de la diversidad de definiciones e interpretaciones que adquiere la extensión universitaria y su praxis. Asimismo, debido a la autonomía con la cual las universidades nacionales y sus comunidades universitarias la interpelan, requiere de ciertas identidades y metodologías que asuman el compromiso de generar condiciones propicias para la extensión universitaria en el siglo XXI. La Red Nacional de Extensión Universitaria (REXUNI) nació al calor del crecimiento exponencial que tuvieron las prácticas y actividades de vinculación comunitaria y de extensión en los últimos años. Esto no se habría dado si no se hubiera tratado de interpretar el espíritu de la Reforma Universitaria de 1918. La singularidad de este acontecimiento que marcó la historia de la universidad pública en nuestro país y en América Latina permite a la REXUNI sugerir a las universidades nacionales una hoja de ruta respecto de los postulados reformistas intrínsecos en la extensión universitaria. Pero también le permite señalar un conjunto de temas que la agenda nacional y latinoamericana de la extensión universitaria no puede dejar de considerar.

Palabras clave

- Reforma Universitaria

- Red Nacional de Extensión Universitaria

- Extensión universitaria

- Agenda nacional de extensión

\section{Resumo}

O artigo a seguir propõe refletir acerca dos trajetos e desafios que tem enfrentado e enfrenta a extensão universitária como função substantiva das instituições de educação superior de gestão pública. Este trânsito é contínuo e fluente, próprio da diversidade de definições e interpretações que adquire a extensão universitária e sua práxis. Ainda, devido à autonomia com que as universidades nacionais e suas comunidades universitárias interpelam-na, requer de determinadas identidades e metodologias que assumam o compromisso de gerar condições propícias para a extensão universitária no século XXI.

A Rede Nacional de Extensão Universitária (REXUNI) nasceu do fervor do crescimento exponencial que tiveram as práticas e atividades de vinculação comunitária e de extensão nos últimos anos. Isto não teria acontecido se não se tivesse tentado interpretar o espírito da Reforma Universitária de 1918. A singularidade deste fato que marcou a história da universidade pública em nosso país e na América Latina permite à REXUNI sugerir às universidades nacionais um roteiro a respeito dos postulados reformistas intrínsecos na extensão universitária. Mas também lhe permite indicar um conjunto de assuntos que a agenda nacional e latino-americana da extensão universitária não pode deixar de considerar.

\section{Palavras-chave}

- Reforma Universitária

- Rede Nacional de Extensão Universitária

- Extensão universitária

- Agenda nacional de extensão

\section{Para citación de este artículo}

Itoiz, J. P. (2017). La interpelación de la Reforma Universitaria a la agenda de la Red Nacional de Extensión Universitaria argentina. Revista $+E$ versión en línea, 7(7), 104-109. Santa Fe, Argentina: Ediciones UNL. 
la universidad debe asumir la responsabilidad de participar, interpretar, aprender y aportar en todos los procesos de cambio social, y en esta tarea la extensión universitaria juega un rol determinante.

\section{Introducción: algunas reflexiones en torno}

\section{a la Reforma Universitaria}

El movimiento reformista ha sido, sin lugar a dudas, el movimiento cultural y educativo más importante e influyente que dio nuestro país y también América Latina. Instaló, entre otros principios, el libre acceso a la educación superior, la democratización del conocimiento, la libertad de cátedra y la extensión universitaria y su rol social.

La extensión universitaria se transformó en estos casi 100 años en la función de las universidades que ha influido de manera más categórica en la formación integral de los estudiantes.

Si bien se parte de la idea de que la universidad tiene como premisa fundamental la producción y la transmisión del conocimiento, no es válido suponer que la tarea de la extensión se lleve a cabo en una sola dirección. La universidad es un espacio de encuentro que genera vínculos con distintos sectores de la sociedad, por lo que el conocimiento fluye en ambos sentidos. Con esta idea, el objetivo es atraer una mayor cantidad de personas a la universidad, por eso necesariamente debemos estrechar los vínculos con la sociedad. La universidad, al relacionarse con la comunidad, promueve la inclusión y la integración social a través de su aporte educativo, cultural y social.

De esta manera, vale la pena recordar qué es lo que entendemos por extensión universitaria.

Se la ha definido y se la puede definir casi permanentemente. Por ejemplo: es la actividad mediante la cual la universidad aporta a la sociedad, en forma crítica e innovadora, los resultados y logros de la docencia y la investigación; y por medio de la cual, al conocer la realidad social y su cultura, se enriquece y redimensiona toda su actividad académica conjunta.

La universidad es un medio de desarrollo social al servicio de la comunidad para contribuir, entre otras cosas, al bienestar de la población, al desarrollo económico sustentable, a la preservación del medio ambiente, al fortalecimiento de la identidad cultural.

Por eso debe interactuar permanentemente con la sociedad, para el relevamiento y análisis de las necesidades y problemáticas de la comunidad y para promover propuestas y alternativas innovadoras. Sin embargo, no siempre quienes hablan de extensión universitaria la definen de la misma manera. Tal vez una explicación a esto pueda ser que, continuamente, la extensión universitaria se va enriqueciendo y reformulando. Desarrolla una amplísima gama de posibilidades y es la herramienta más poderosa con la que cuentan las universidades públicas para hacer realidad su inserción social. Se ha transformado en un verdadero factor de integración social. A su vez, la extensión universitaria se ha convertido en un espacio de participación para la construcción del conocimiento de una manera amplia y abierta, compartida.

Por lo tanto, definir la extensión universitaria en el centenario de la Reforma implica un doble desafío. Por un lado, dar cuenta del impacto social que las intervenciones universitarias han provocado y provocan en la comunidad. Por otro lado, evitar caer en una definición reduccionista, que refleje exclusivamente el debate que se da en el seno de la comunidad universitaria, y que se transforme en una simple declaración de principios o de deseos.

La diversidad de significados, prácticas y procesos, es la principal fortaleza de la extensión universitaria. Por eso, cuando hablamos de extensión, debemos hacerlo desde una perspectiva integral e integradora.

A partir de 1918, los principios reformistas se desplegaron en las universidades argentinas y luego se extendieron a Latinoamericana; durante el transcurso del siglo $\mathrm{XX}$, se irradiaron a otras regiones del mundo hasta que, en 1998, en la Cumbre Mundial de UNESCO se consagró su aceptación universal.

Desde aquellos primeros años, la universidad ha cambiado porque el contexto social en el que está inmersa también se fue modificando. Hoy en día, los cambios no solo son necesarios, son además inevitables. En consecuencia, la universidad debe asumir la responsabilidad de participar, interpretar, aprender y aportar en todos los procesos de cambio social, y en esta tarea la extensión universitaria juega un rol determinante. 


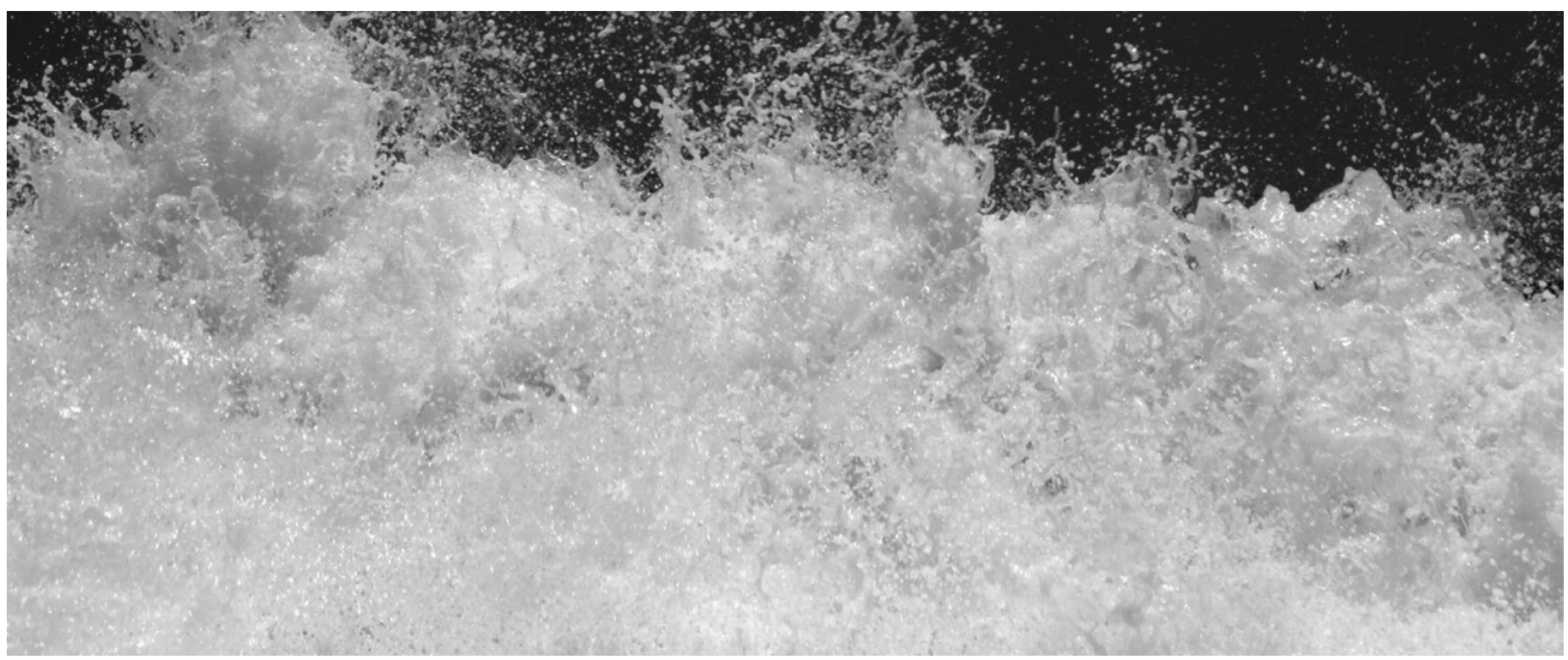

(c) Laura Hormaeche

Del mismo modo, la relación universidad-sociedad impone nuevos desafíos, como, por ejemplo, articular el sistema universitario para que interactúe con el Estado y con la comunidad para el desarrollo del país así como formar ciudadanos responsables, con compromiso social, con valores democráticos y humanísticos capaces de adaptarse a la dinámica de un mundo en constante cambio. A lo largo del tiempo, y teniendo en cuenta la autonomía de las universidades, la extensión universitaria ha ofrecido y ofrece una diversidad de posibilidades de trabajo que no comprenden las demás funciones de la universidad.

Sus prácticas y actividades pueden desarrollarse mediante:

- Actividades de transferencia al entorno comunitario de los conocimientos generados por la universidad, ya sea mediante conferencias, jornadas, seminarios, charlas, talleres, etcétera.

- Actividades de capacitación y formación continua, que permitan el acceso al conocimiento, el intercambio de experiencias y la actualización profesional y laboral.

- Actividades culturales, artísticas y deportivas, mediante programas y proyectos culturales, de divulgación científica, a través de programas para públicos específicos, como son los programas para adultos mayores, para niños y adolescentes.

- Prácticas comunitarias que asumen la responsabilidad de trabajar por la transformación social mediante la formación de ciudadanos y profesionales con espíritu crítico y comprometido, en espacios de aprendizaje e intercambio de saberes y articulando la enseñanza con la extensión.

- Proyectos de extensión universitaria que estimulan y fomentan las actividades y acciones de extensión en un marco multidisciplinario, de modo de afianzar los vínculos con la comunidad.
En todas las universidades nacionales los programas de extensión tienen como meta crear nuevos espacios de intercambio de conocimientos.

Las actividades de extensión universitaria han sido un baluarte en la consolidación de los proyectos institucionales de las universidades y en estrechar el vínculo universidad-sociedad, destacándose no solo por la formación de profesionales sino también por entender a la educación como un derecho y como un aprendizaje que se extiende a lo largo de toda la vida.

La jerarquización de la extensión debe incluir tres dimensiones en las cuales debemos trabajar.

En primer lugar, el reconocimiento académico de la función de extensión, incorporando a la normativa que contempla, regula y valora, la función de extensión en las trayectorias de los miembros de la comunidad universitaria: docentes, estudiantes, graduados y no docentes.

En segundo lugar, la inserción curricular de la extensión a través de propuestas académicas de formación y capacitación al respecto, ya sea de grado o de posgrado, que reconozcan las experiencias extensionistas por medio de la asignación de créditos académicos. Y en tercer lugar, el reconocimiento presupuestario de la función de extensión.

Pero estas dimensiones no deben estar desligadas de la cuestión de la calidad universitaria. La jerarquización de la extensión universitaria no puede transitar un camino separado de la calidad de sus prácticas, de sus programas y de sus actividades.

Resulta imperioso para la extensión universitaria avanzar hacia modelos de gestión de calidad, centrándonos en hacer bien aquello que es esencial para el desarrollo de la función de extensión. 


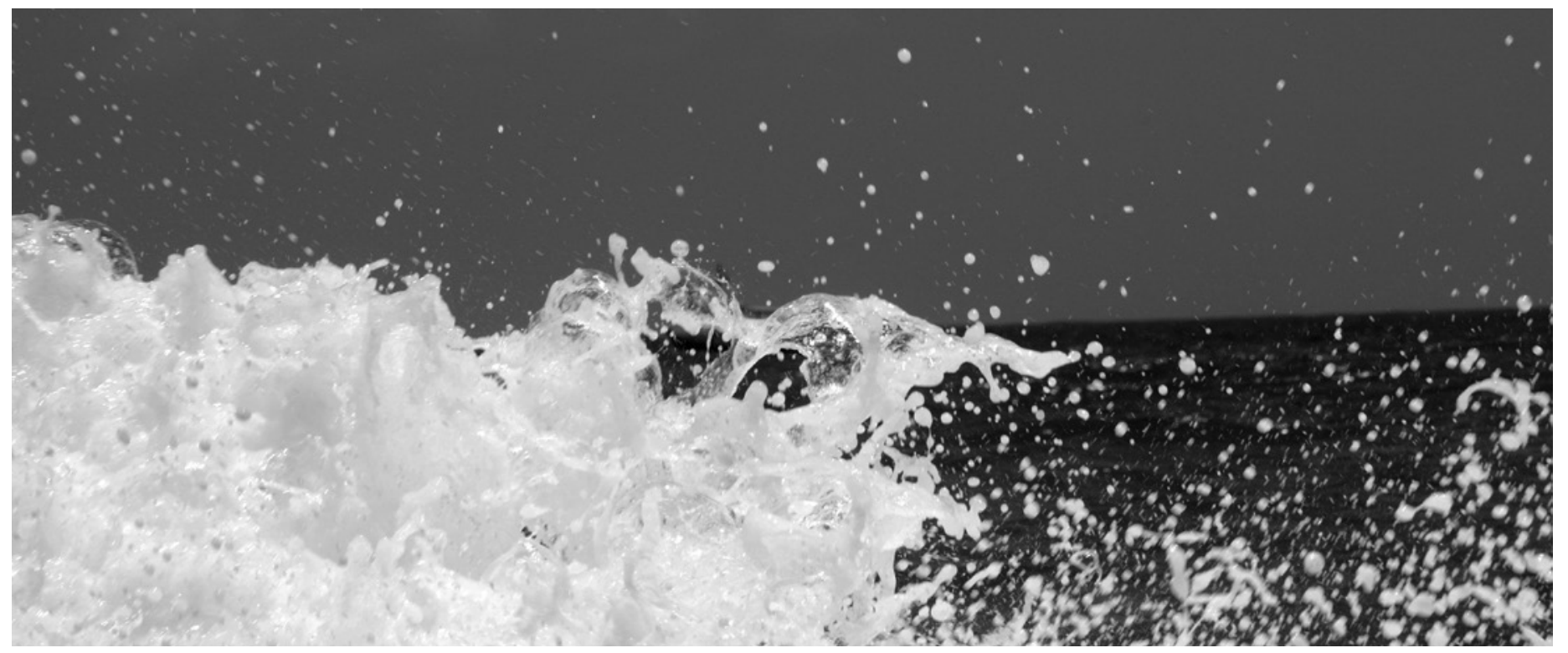

(C) Laura Hormaeche

En este sentido, la calidad de las actividades universitarias debe estar puesta al servicio del compromiso social de la universidad, con el fin irrenunciable de promover mediante la educación la movilidad social ascendente y el mejoramiento de la calidad de vida de los ciudadanos.

En la actualidad, las tensiones sociales, la desigualdad, la defensa de los valores democráticos y de los derechos humanos, como también la integración educativa, son cuestiones que se evidencian en la Argentina y también en América Latina. En este escenario, la extensión universitaria tiene un rol preponderante que cumplir, el cual debe manifestarse a través de la política, dotar de un espacio institucional al conocimiento y reafirmar el derecho a la educación como un derecho humano. Se requiere que trabaje y se vincule con los actores sociales y comunitarios, provoque la participación de los estudiantes en sus prácticas sociales educativas y trate de impulsar la integralidad de las funciones de la universidad: la docencia, la investigación y la extensión.

Si se trabaja en este sentido, se podrá construir una universidad abierta, integradora y socialmente comprometida para continuar el camino iniciado hace un siglo por la Reforma Universitaria.

A la vez, los universitarios debemos trabajar para que la extensión universitaria sea cada vez más visible, no solo hacia el interior de la academia sino también en la consideración de la ciudadanía.

\section{La Agenda de la REXUNI}

En los últimos años, el debate sobre el funcionamiento del sistema universitario ha adquirido gran relevancia, ya que es una coincidencia generalizada que ningún país puede aspirar a alcanzar índices razonables de desarrollo y de calidad de vida para sus ciudadanos si no fortalece su sistema educativo.

Desde las universidades públicas, la organización de la actividad académica, las cuestiones vinculadas a la investigación y transferencia, como las más diversas acciones y actividades de la extensión, inciden en el medio social y nos proponen un debate permanente.

Ese debate no debe ser enclaustrado sino todo lo contrario, tiene que ser abierto a la comunidad puesto que esta es la que sostiene, con su trabajo, al sistema público de educación al cual deben estar dirigidos todos los esfuerzos para que la educación y la cultura sean la solución a la exclusión y a la desigualdad.

La expansión de la educación superior ha permitido la incorporación de miles de estudiantes que de otro modo no hubieran podido llegar a la universidad y esto, de alguna manera, ha dado a las universidades un reconocimiento social y un prestigio del que no gozan otras instituciones de nuestro país.

A su vez, esta percepción social de las universidades ha elevado la vara y hoy las circunstancias y los hechos nos ponen, a los universitarios, en un reto de dar cumplimiento a esta premisa, pensando y llevando a la práctica una universidad que brinde una formación integral y que esté asociada a la calidad y la eficacia de la actividad universitaria.

Esta oportunidad coloca a la extensión universitaria en posición de dar respuestas a las expectativas y de colaborar en la construcción de una sociedad más igualitaria.

El puntapié inicial estuvo asociado a la idea de consolidación de un sistema de educación superior inclusivo y de calidad, que tuviera a la extensión universitaria como una herramienta 
para facilitar la formación integral, pilar fundamental de una educación pensada en clave de movilidad social e igualdad de oportunidades. Y el nacimiento de la REXUNI en el año 2007 hizo posible que las actividades, programas y proyectos de extensión que venían ejecutando las universidades nacionales desde hace años comenzaran a tomar visibilidad y difusión en el sistema universitario de la educación pública.

Hoy la agenda de la REXUNI pasa por perfeccionar el camino transitado y explicitado mediante el Plan Estratégico de la Extensión (Acuerdo Plenario del Consejo Interuniversitario Nacional $N^{\circ}$ 811/12), que contextualizó a la extensión universitaria como parte de una educación entendida como un bien social y un derecho universal al expresar que:

"La extensión es una función sustantiva que integrada con la docencia y la investigación forman parte de un modelo de universidad que caracteriza al sistema universitario nacional. La extensión —desde su dimensión académica, dialógica, pedagógica y transformadora - le confiere a la propia universidad la posibilidad de "mirar" de manera crítica y permanente sus propias prácticas académicas y repensar sus políticas institucionales. Las políticas de Extensión ubican a la universidad en diálogo permanente con las organizaciones de la sociedad civil y el Estado, contribuyendo a partir del conocimiento y el pensamiento crítico, al estudio, diseño, formulación, monitoreo y evaluación de políticas públicas en la búsqueda de una mayor y mejor calidad de vida para todos sus habitantes". (CIN, 2012:s/n)

Fundamentalmente, son tres las líneas estratégicas que se destacan: 1. Internacionalización de la extensión universitaria: basándose en la cooperación y en la comprensión de la diversidad de los sistemas educativos, como conceptualizaciones para entender las diferentes realidades, que fortalezcan el compromiso de una mayor integración regional y la vinculación internacional, privilegiando las relaciones con los sistemas de educación superior de América Latina y el Caribe.

Como bien lo señala el Plan Estratégico,

"es necesario trabajar en la cooperación a nivel internacional, que promueva el desarrollo y la calidad institucional, así como la complementación de las capacidades para fortalecer las funciones sustantivas. A partir de la interpelación a aquellos contextos de cooperación regional, que a su vez incorpora constantemente nuevas dimensiones, y con el objeto de fortalecer la función Extensión”. (CIN, 2012:s/n)

La REXUNI debe promover el intercambio entre universidades latinoamericanas, caribeñas y europeas a través de programas, proyectos y acciones de extensión, con el propósito de difundir y reforzar el compromiso social de la educación superior.
Las experiencias de los últimos años reflejadas en la expansión de la Unión Latinoamericana de Extensión Universitaria (ULEU), y en el trabajo de las universidades que participan de la Asociación de Universidades del Grupo Montevideo (AUGM), dan muestra de las enormes posibilidades de trabajo que existen en nuestro continente, como también de las posibilidades de expansión hacia otras latitudes respecto de la extensión universitaria y su desarrollo en nuestro país.

2. Promoción de la inserción curricular de la extensión universitaria: Las prácticas educativas que se desprenden de la extensión deben estar insertas en la dinámica pedagógica de la universidad. Dichas prácticas plantean incorporar al trayecto académico (currícula) del estudiante universitario diversas modalidades en el campo de la educación experiencial al desarrollo de acciones en campo (fuera del aula, en el territorio) que involucren las tres funciones sustantivas de la educación superior, docencia, investigación y extensión. Este tipo de prácticas se lleva a cabo en diálogo con organizaciones sociales e instituciones públicas, desde la identificación misma del problema a la ejecución de la propuesta. Asimismo, docentes e investigadores tienen una participación activa en el desarrollo de las prácticas, y pueden sumarse también graduados y personal de apoyo académico. La incorporación a la currícula de estas prácticas pretende sumar contenido a la formación profesional de los futuros egresados, orientándolos hacia una actitud comprometida y crítica respecto de la realidad (CIN, 2012).

Para ello, la REXUNI debe proponerse la divulgación de las iniciativas que en este sentido llevan adelante las universidades nacionales, para que sirvan de ejemplo a las nuevas universidades que están iniciando este camino. La colaboración, la cooperación interinstitucional y el intercambio de experiencias son fundamentales que pensar un sistema en permanente evolución, donde se pueda incrementar la cantidad de prácticas de incorporación curricular. A su vez, la Red Nacional debe aunar esfuerzos y desarrollar acciones conjuntas con las áreas académicas de las universidades que posibiliten la incorporación curricular de la extensión, tales como: formación docente; construcción de indicadores de evaluación de estas prácticas; reconocimiento institucional en normativas vinculadas con carrera docente y con créditos académicos.

3. Creación de sistemas de programas y proyectos de extensión en las universidades nacionales con el objetivo de expandir y reconocer las actividades de extensión a nivel nacional: Este tipo de sistemas permite dar definiciones institucionales sobre los campos o temas de interés que la universidad identifica y vincula con las problemáticas sociales y comunitarias para ser incorporadas a la agenda pública. Los proyectos de extensión representan espacios de trabajo con el Estado en sus diferentes jurisdicciones y con los diversos actores de la comunidad y abordan las más diversas y complejas problemáticas sociales y productivas en un territorio determinado. 
Cada proyecto se estructura de forma tal que asegura que sus objetivos sean factibles de alcanzar. Los mismos cuentan con una planificación y metodología que definen todo el proceso, la organización, las demandas, los actores intervinientes, los recursos humanos y económicos disponibles y su optimización. Cada proyecto de extensión forma parte de un proceso de intervención social e implica propuestas de desarrollo cultural, social o productivo, que brindan aportes a la solución de problemas concretos. Esta intervención, interactiva y dialógica, pone en juego los conocimientos científicos aportados por la universidad con los saberes y conocimientos de la comunidad (CIN, 2012).

La REXUNI debe perseverar en la promoción de estos sistemas porque, además de la necesidad de que cada vez más universidades cuenten con ellos, son un instrumento de recolección de información fundamental para la elaboración, la gestión, la evaluación y la sistematización de las políticas de extensión de calidad. Estas políticas deben apuntar a mejorar las condiciones para el surgimiento de nuevos programas y espacios multidisciplinarios, vincular la docencia, la extensión y la investigación y favorecer las condiciones para que la extensión universitaria sea parte integral del proceso educativo, de una manera directa y activa en su vinculación con su entorno social.
Y en vísperas de cumplirse los 100 años de la Reforma Universitaria, esta Red debe tener más presente que nunca que la incorporación de la extensión universitaria como función sustantiva fue un producto del movimiento reformista.

Retomemos ese espíritu y, más allá de los escollos o dificultades, redoblemos los esfuerzos y el compromiso de llevar plenamente los conocimientos universitarios a la sociedad.

Solo si promovemos y jerarquizamos a la extensión universitaria como parte de nuestras actividades cotidianas, desde el lugar que nos brinda la universidad, cumpliremos con nuestra responsabilidad como institución de educación pública.

Tenemos que asumir el compromiso innegociable de trabajar a favor de una educación que esté al servicio del desarrollo y que brinde a todos aquellos que quieran hacer el esfuerzo de estudiar los instrumentos necesarios para una vida mejor.

\section{Referencias bibliográficas}

CIN (2012). Acuerdo Plenario N1 811. Plan Estratégico de la Extensión. Consejo Interuniversitario Nacional. Buenos Aires. 